\title{
sciendo
}

Transport and Telecommunication, 2020, volume 21, no. 1, 32-46

Transport and Telecommunication Institute, Lomonosova 1, Riga, LV-1019, Latvia

DOI 10.2478/ttj-2020-0003

\section{MOBILITY AWARE NETWORK SELECTION IN A HETEROGENEOUS WIRELESS ENVIRONMENT}

\author{
Fayssal Bendaoud ${ }^{1}$, Marwen Abdennebi ${ }^{2}$, Fedoua Didi ${ }^{3}$ \\ ${ }^{1}$ LabRI-SBA Lab, Ecole supérieure en Informatique, Sidi Bel Abbes, Algeria \\ ${ }^{2}$ L2T1 Lab, University of Paris 13, France \\ ${ }^{3}$ LRIT Lab, University of Tlemcen, Algeria
}

\begin{abstract}
Different Radio Access Technologies (RATs) coexist in the same area has encouraged the researchers to get profit from the available networks by selecting of the best RAT at each moment of the call session to satisfy the user requirements. In this paper, we address a real-world problem which is the frequent mobility of the users in heterogeneous networks. We present in this paper a framework that allows users to select the best networks for the whole call session especially form a mobility perspective. The framework consists of several steps, starting by the path prediction which is performed using a Markov model order 2. The second step is to make the network selection on the zones of each predicted path, while in the third step; we get the best RAT's configuration for each predicted path. Finally, we use another function to select one of the best configurations to be used for all the possible used paths. The results show that our proposal performs very well by eliminating the unnecessary vertical handover while maintaining a good Quality of Service (QoS).
\end{abstract}

Keywords: Radio Access Technologies, network selection, paths prediction, Markov model, vertical handover, QoS, call session

\section{Introduction}

The next generation of wireless network is characterized by the coexistence of various wireless access networks such as, the basic WLAN IEEE802.11 family, UMTS, HSPA and the LTE The heterogeneous environment resides in the different radio access links. This heterogeneous system (Bendaoud et al., 2018) has different characteristics such as, network availability, coverage and Quality of Service (QoS). The main difference between the different RATs is related to the speed of movement of the mobile node. One of the fundamental ideas of the heterogeneous systems consists to give the users the ability to choose the best network among multiple existing RATs based on several criteria, this process is called the Network Selection (NS) (Yafang et al., 2010; Bari. and Leung, 2007).

The network selection procedure consists in selecting the best available network among those accessible (Bendaoud et al., 2015). The best network differs from one user to another due to the various parameters (preferences) involved in the process such as cost, QoS, energy consumed and even mobility (Lahby et al., 2015; Fu et al., 2010)

Mobility is the key feature of wireless networks that differentiates them from the wired ones. In the same time, the movement of the mobile user is the major cause for the QoS deterioration since the user might change the access point or even the network rapidly. Ensuring the required QoS for the mobile terminal is a very important field of research (Shaikh, 2010) which is the scope of this paper. In the following, we propose to study the mobility of users and the network selection within a heterogeneous environment. In particular, when a mobile terminal comes out of the coverage area of an access point in which it was connected, this situation is directly related to the physical mobility of the mobile user which might generate an interruption of connection when performing a vertical handover. To avoid these probable interruptions, the operator must take steps in advance to minimize the number of vertical handover especially the unnecessary ones. These interruptions might generate packet losses, transmission delay and even increase the energy consumption. So, to eliminate these loses, we propose that the operator predicts the paths that are susceptible to be taken by the user with a certain probability using Markov model order 2. The path prediction allows us to take proactive measures to reduce the degradation of QoS due to the unnecessary vertical handover. In this work, we consider the heterogeneous network including (Wi-Fi, 3G+, H+ and LTE).

The main objective of this paper is the adaptation of the network selection process in the case of frequent mobility of the users. The idea is to predict the paths that will be taken by the user with certain probabilities. After that, the operator makes an off-line network selection process considering the zones 
that constitutes the predicted paths. The third step consists in finding the best configuration of RATs for each predicted path after making the off-line network selection. Finally, the operator chooses the configuration of RATs that has the largest mean value for all the paths. This RAT configuration' is the most likely the best suited solution to satisfy the users QoS requirements for the entire call session.

The rest of the paper is organized as follows Section 2 presents the related work whereas in Section 3, we present our proposed approach, we discuss the Markov model used to predict the paths and we present the network selection algorithm used. After that, we give the methodology which allows us to reduce the vertical handover occurrences in the context of mobility while maintaining the required QoS. In Section 4, we provide an example to explain how our proposal does work; we have also compared theoretically the performance of the proposed approach with some other works. We present concluding remarks in Section 5.

\section{Related work}

In this section, we present the works in the literature that treat the mobility issue, the prediction of states, the network selection and the vertical handover.

In (François and Leduc, 2006) the authors made a comparison between intrinsic and the extrinsic methods (prediction made by the mobile user or by the Access point) based on a Markov model to predict the states of the mobile user. This work concluded that the intrinsic approach is more accurate and produced a higher performance. In (Song et al., 2004) authors have compared the Markov model with other predictors like LZ predictors. They produced results showing that the Markov model is more accurate and suitable. After that, they proved that a Markov model order 2 is the most beneficial amongst the Markov models order k withk $\in$. In (Savitha and Chandrasekar, 2011a) and (Nguyen-Vuong, 2008) the authors have used the Simple Additive Weight method SAW to get a ranked list of networks, while in (Salih et al., 2015) the authors made a mix between game theory and SAW method. The main benefits of SAW are its simplicity and low complexity. On the other hand, it has two major drawbacks, first: a parameter can be outweighed by another one, second: the rank reversal phenomenon which represents a problem for the entire MADM approach. Furthermore in (Savitha and Chandrasekar, 2011a), the authors have compared the performances of the vertical handover VHO using SAW and TOPSIS. The authors conclude that the TOPSIS method outperforms the SAW method. In general, we can say that the compensatory methods such as TOPSIS can avoid the problem where a parameter value can be overshadowed by another one. This is due to the usage of some trade-off between the criteria. This means that a poor value in one criterion is neglected due to a good value in the other criteria. This concept provides more credibility for whole NS process in comparison to the non-compensatory methods, which use the thresholds and do not permit any kind of trade-off between criteria. A comparative study between SAW and WPM in the context of vertical handover is done in (Savitha and Chandrasekar, 2011b). The authors use the relative standard deviation as a metric of comparison and they obtained a conclusion that WPM is better than SAW in some cases. In (TalebiFard and Leung, 2011) the authors use the WPM method in the context of heterogeneous systems. Their conclusion states that the WPM method is a more robust approach for dynamic decision making while it penalizes the attributes with poor quality to a greater extent. The authors of (Gambs et al., 2012) have proven that Markov chain order 2 gives more prediction accuracy in the ranges of $701 \%$ to $951 \%$. They also explained that using Markov model order $\mathrm{k}$ with $\mathrm{k}>2$ does not seem to bring an important improvement at the cost of a significant overhead in terms of computation and memory space for the learning and the processing of the mobility model. The authors in (Fettouh et al., 2013) have made experiments on vertical handover between WLAN and GPRS. They mentioned that the vertical handover latency is a sum of the detection time, the configuration time and the registration time. The latency values presented show that multiple vertical handover can degrade the QoS, especially the unnecessary ones. In (Magagula et al., 2010), the authors showed that mobile IPV6 for Fast Handover is the suitable protocol to satisfy the QoS requirements. The obtained simulation results show that the mechanism reduces the vertical handover delay and packet loss better than ordinary PMIPv6 and FMIPv6. However, there is a slight trade-off in signalling overhead as the number of vertical handover per time unit is increased when compared to ordinary PMIPv6. In (Hasib, 2006), the authors have proposed an uplink scheduler algorithm; they evaluated their proposal with various mobility schemes. Two user profiles have been tested and it was shown that the end-to-end delay can be reduced if a parallel transmission profile is chosen. In this study the authors have used several 802.11n access points which it doesn't represent a real heterogeneous system. The proposed scheme is based on two notions: the queue and the priority of the packets. It is known that the priority based systems are not suitable especially when using heterogeneous networks. Because, different RATs means different packets 
structures; thus, they cannot be treated with the same way. Consequently, the proposed method is interesting but it doesn't treat the real case of heterogeneous networks. In (Nguyen-Duc and Kamioka, 2016) the authors have studied the usage of mobility-related factors for network selection in HWNs and provided a scheme to use them in the same way as other factors in a MADM based generic framework. The authors used the MADM method to rank the alternatives, the AHP method to get the weigh factors and then considered nine criteria: the average handover cost, monetary cost, bandwidth, power consumption, security level, bit error rate, burst error rate, jitter, traffic load and signal strength. Though the proposed work took in consideration multiple criteria, several of them are strongly correlated such as the bandwidth and the traffic load, the bit error rate is related to the burst error rate. So, there are some useless criteria. Regardless of the correlated parameters, considering nine parameters increases the complexity of this approach significantly which can delay the NS process. Hence, introducing further unnecessary communication delay. The second problem is, no criterion is representing the mobility. The mobility is a random concept which cannot be modelled with a static factor in a system. The authors conclude by saying that they demonstrated that mobility-related factors should be considered for network selection, and that their scheme achieved the goal of selecting the appropriate network for the mobile terminal with various mobility features. In our opinion, this is not necessarily true because of the random nature of the mobility. Authors in (Wang and Binet, 2009) have proposed a dynamic management of vertical handover with advanced resources reservation. This feature is done by determining the future localisation of the mobile terminal. The authors have considered only the WLAN and the UMTS without presenting a real and clear concept to make the selection between the networks.

In this work, we will try to get profit from all these ideas and combined them to get a novel approach for Network Selection suited for high mobility. Of course our approach is a probabilistic one and it is based on the prediction of the path that will be used by the user. It will consider all the potential paths and gives the best configuration of RATs to provide user with the best QoS.

\section{The proposed scheme}

This section represents the main part of this work. Indeed, the objective of this work is to provide the mobile user with the best QoS during the call session. The primary reason of QoS degradation is the motion of the users, the existing works try to grant users the requested QoS instantly without ensuring the continuity of this QoS during the call session. To attain our objective, we start with the prediction of path with some probabilities. We use these probabilities to estimate the geographical path (in term of cells) that it will be taken by the mobile user. The second step is to make the network selection on the predicted paths to classify the RATs at each state of the predicted paths. The third step consists in collecting all the configurations (the initial and alternative, more details see sections below) of RATs that represents all the paths predicted. The final step is using the mean value formula to decide which configuration to use for all the paths. The proposed solution consists of 4 steps; the first 2 steps are the most important which are the path prediction and the network selection on the paths predicted. The second step finishes by providing a list of ranked RATs. The succession of the RATs having the same ranking order on each zone constitutes a configuration. We have added two additional steps 3 and 4 to optimize the vertical handover occurrence in each configuration.

\subsection{Path prediction}

We are interested in the on-line predictors, which examine the user mobility history, extract the current context, and predict the next location. Once the next location is known, the history is updated and the next context is added to the history. Then, the predictor prepares to extract the next location and so on. Basically, two famous families of predictors exist: the order-k Markov predictors and the LZ-based family. In (Song et al., 2004) the authors compare the two families and give the following conclusions:

- The LZ-based family has the same performance with the order-1 Markov model.

- The order-2 Markov process outshines the order-1 process.

- The order-2 Markov predictors worked as well as or better than the more complex compressionbased predictors, and better than the higher order Markov predictors. Particularly, O (3) (or above) Markov predictors doesn't provide improves over O (2) Markov since it reduces the accuracy by failing to make predictions most of the time.

So, in the following, we explain the Markov process theoretically, we give the mathematical description and we present an example to understand the whole process and how we apply our approach in a real world scenario. A Markov process is a stochastic process based on the Markov property, this 
means that the useful information for the prediction of the future is completely contained in the present state of the process and it doesn't depend on previous states. Hence, the system is called memory-less (Roman et al., 2016), Equation 1.

It was proven in (Song et al., 2004) and (Gambs et al., 2012) that the order 2 Markov models give more accurate predictions than simple Markov chains. Accordingly, in our work, the path prediction is done using the Markov model order 2. The prediction models are used in several domains such as, the prediction of weather conditions, chess games, poker ...etc. In this paper we are going to use this model to predict the movement of the mobile users. There are plenty of previous studies that used the Markov model to predict the path of users (Wang and Binet, 2009; François and Leduc, 2006). However, in addition to path prediction, our methodology introduces three other steps to provide the users with a successful and efficient selection of networks to ensure that the users are best served during the call session.

$\boldsymbol{P}=\boldsymbol{P}\left(\boldsymbol{L}_{\text {next }} \mid \boldsymbol{L}_{\text {current }}\right)$

The order- $k$ Markov predictors assume that the next location can be predicted from the sequence of the $k$ most recent symbols in the location history (Song et al., 2004). At each instant, a user has a unique location, i.e., is connected with a single base station. The location is formalized as a string representing the history of locations where the user was/is connected. The set of all possible locations is noted ' $A$ ', one location from ' $A$ ' is ' $a$ '. Each user has a set of visited (precedent) locations, $\boldsymbol{L}_{\boldsymbol{n}}=\boldsymbol{L}_{\mathbf{1}} \ldots \boldsymbol{L}_{\boldsymbol{n}}$. The stochastic Markov process order $k$ is:

$P\left(L_{i=a} \mid L_{1} \ldots L_{i-1}\right)=P\left(L_{i=a} \mid L_{i-k} \ldots L_{i-1}\right)$,

$\forall \boldsymbol{a} \in \boldsymbol{A}, \boldsymbol{i}, \boldsymbol{k}<n$.

$\boldsymbol{L}_{\boldsymbol{i}}$ means the location at an instant ' $\mathrm{i}$ '. The distribution of the possible next locations can easily be learned on-line. It is assumed that the prediction agent is regularly notified by the movement of the mobile user, the efficiency of the prediction is correlated with the notification periods. $\boldsymbol{L}^{\boldsymbol{m}}$ is the location history of the mobile $\mathrm{m}$, the estimation formula of order-n Markov model is:

$P\left(L_{i=a} \mid L_{1} \ldots L_{i-1}\right)=\frac{o\left(L_{i-n}^{m}, \ldots, L_{i-1}^{m}, a ; L^{m}\right)}{O\left(L_{i-n}^{m}, \ldots, L_{i-1}^{m} ; L^{m}\right)}$.

Where $O(a ; B)$ is the number of times the substring ' $\mathrm{a}$ ' occurs in the string $\mathrm{B}$. These values constitute the transition matrix. The location history is a sequence of location observations, for example, the user's location recorded once every ten seconds where, the $\boldsymbol{a}_{\boldsymbol{i}} \neq \boldsymbol{a}_{\boldsymbol{i}+\mathbf{1}} 0<\mathrm{i}<\mathrm{n}$. The predictor uses the location's history $L$ as a sequence of abstract symbols without interpreting them.

To explain how the Markov process is applied, we propose a simple example: We consider an environment associated with a location history $\mathrm{L}=$ "ADGHEFCBABCFHGEDABCFHGDABCEHGDADGDEHFCBA" (see Figure 1). Assuming that the mobile user is in zone ' $A$ ' at time $\boldsymbol{t}_{\mathbf{0}}$, after previously being in an arbitrary zone $\boldsymbol{\alpha}$ (previous location). Next, we will explain how the Order-2 Markov process works, especially how to extract the path's probabilities. We suppose that the mobile user can stay in the same zone with certain probability which is fixed to 0.1

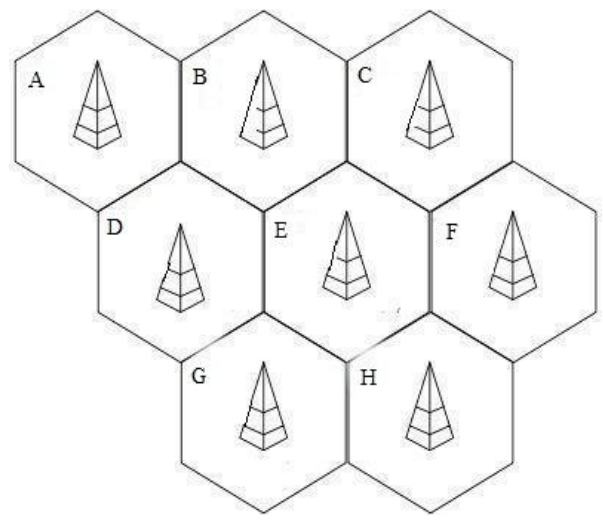

Figure 1. Example of a zone map 
Based on the formula (3), the part $\boldsymbol{L}_{\boldsymbol{i}-\boldsymbol{n}}^{\boldsymbol{m}}, \ldots, \boldsymbol{L}_{\boldsymbol{i}-\mathbf{1}}^{\boldsymbol{m}}$ of the formula is known for the Markov process, in this example at the first step, it is equal to ' $\alpha A$ ', and the process will try to find the ' $a$ ' part which is the next location.

Table 1 contains the entire process and shows how to get the paths and their probabilities.

Table 1. The order 2 Markov process

\begin{tabular}{|c|c|c|c|c|c|c|c|}
\hline Round & C & $\mathbf{O}(\mathbf{c} ; \mathrm{L})$ & $\mathbf{a}$ & $O(c a ; L)$ & $\mathbf{P}($ ca) & Sub-path & P(sub-path) \\
\hline 1 & $\begin{array}{l}\alpha A \\
\alpha A\end{array}$ & 0.36 & $\begin{array}{l}\text { B } \\
\text { D }\end{array}$ & $\begin{array}{l}3 \\
2\end{array}$ & $\begin{array}{l}3 / 5 \\
2 / 5\end{array}$ & $\begin{array}{l}\alpha A \mathrm{~B} \\
\alpha A \mathrm{D}\end{array}$ & $\begin{array}{l}0.54 \\
0.36\end{array}$ \\
\hline 2 & $\begin{array}{l}\mathrm{AB} \\
\mathrm{AD}\end{array}$ & $\begin{array}{l}3 \\
2\end{array}$ & $\begin{array}{l}\mathrm{C} \\
\mathrm{G}\end{array}$ & $\begin{array}{l}3 \\
2\end{array}$ & $\begin{array}{l}1 \\
1\end{array}$ & $\begin{array}{l}\alpha A \mathrm{BC} \\
\alpha A \mathrm{DG}\end{array}$ & $\begin{array}{l}0.54 \\
0.36\end{array}$ \\
\hline 3 & $\begin{array}{l}\text { BC } \\
\text { BC } \\
\text { DG } \\
\text { DG }\end{array}$ & $\begin{array}{l}3 \\
3 \\
2 \\
2\end{array}$ & $\begin{array}{l}\mathrm{F} \\
\mathrm{E} \\
\mathrm{H} \\
\mathrm{E}\end{array}$ & $\begin{array}{l}2 \\
1 \\
1 \\
1\end{array}$ & $\begin{array}{l}2 / 3 \\
1 / 3 \\
1 / 2 \\
1 / 2\end{array}$ & $\begin{array}{l}\alpha A \mathrm{BCF} \\
\alpha A \mathrm{BCE} \\
\alpha A \mathrm{DGH} \\
\alpha A \mathrm{DGE}\end{array}$ & $\begin{array}{l}0.36 \\
0.18 \\
0.18 \\
0.18\end{array}$ \\
\hline 4 & $\begin{array}{l}\mathrm{CF} \\
\mathrm{CE} \\
\mathrm{GH} \\
\mathrm{GE}\end{array}$ & $\begin{array}{l}2 \\
1 \\
1 \\
1\end{array}$ & $\begin{array}{l}\mathrm{H} \\
\mathrm{H} \\
\mathrm{E} \\
\mathrm{H}\end{array}$ & $\begin{array}{l}2 \\
1 \\
1 \\
1\end{array}$ & $\begin{array}{l}1 \\
1 \\
1 \\
1\end{array}$ & $\begin{array}{l}\alpha A \mathrm{BCFH} \\
\alpha A \mathrm{BCEH} \\
\alpha A \mathrm{DGHE} \\
\alpha A \mathrm{DGEH}\end{array}$ & $\begin{array}{l}0.36 \\
0.18 \\
0.18 \\
0.18\end{array}$ \\
\hline 5 & $\begin{array}{l}\mathrm{FH} \\
\mathrm{EH} \\
\mathrm{HE} \\
\mathrm{EH}\end{array}$ & $\begin{array}{l}1 \\
1 \\
1 \\
1\end{array}$ & $\begin{array}{l}\mathrm{G} \\
\mathrm{C} \\
\mathrm{F} \\
\mathrm{F}\end{array}$ & $\begin{array}{l}1 \\
1 \\
1 \\
1\end{array}$ & $\begin{array}{l}1 \\
1 \\
1 \\
1\end{array}$ & $\begin{array}{l}\alpha A \mathrm{BCFHG} \\
\alpha A \mathrm{BCEHC} \\
\alpha A \mathrm{DGHEF} \\
\alpha A \mathrm{DGEHF}\end{array}$ & $\begin{array}{l}0.36 \\
0.18 \\
0.18 \\
0.18\end{array}$ \\
\hline
\end{tabular}

- $\quad c$ : is the concatenation of the current zone and the past zone, the order-2 Markov process. As its name indicates, this process needs 2 previous states to predict the next one.

- $O(c ; L)$ : is the number of times that the c occurs in the location history L.

- $\quad a$ : is the next state, the state that we want to predict.

- ca: is the concatenation between the values ' $c$ ' and ' $a$ '.

- $\quad P(c a)$ : the probability of ' $c a$ ' which is equal to $O(c a ; L) / O(c ; L)$

- $\quad P$ (Sub-path): the probability of the sub-path, the path is a succession of zones predicted from the first round (the zones are represented by alphabetic letters). The probability of the subpath is equal to the product of the probabilities of the predicted zone with the probability of the previous sub-path (the sub-path of the previous round).

The Markov process presented can be modelled by the scheme in Figure 2. This schematic representation of Table 1 shows the order-2 Markov transition from a state to another. It starts with a common state, and it discovers the next states using the location history. The values of the transitions are the probabilities of moving from the current state to the next state.

We remind that, we began from an arbitrary state and we use the location history L with the formula 3 to calculate the probabilities of the transitions. Finally, the probability of a path is the mathematical multiplication of the probabilities of the transitions in this path.

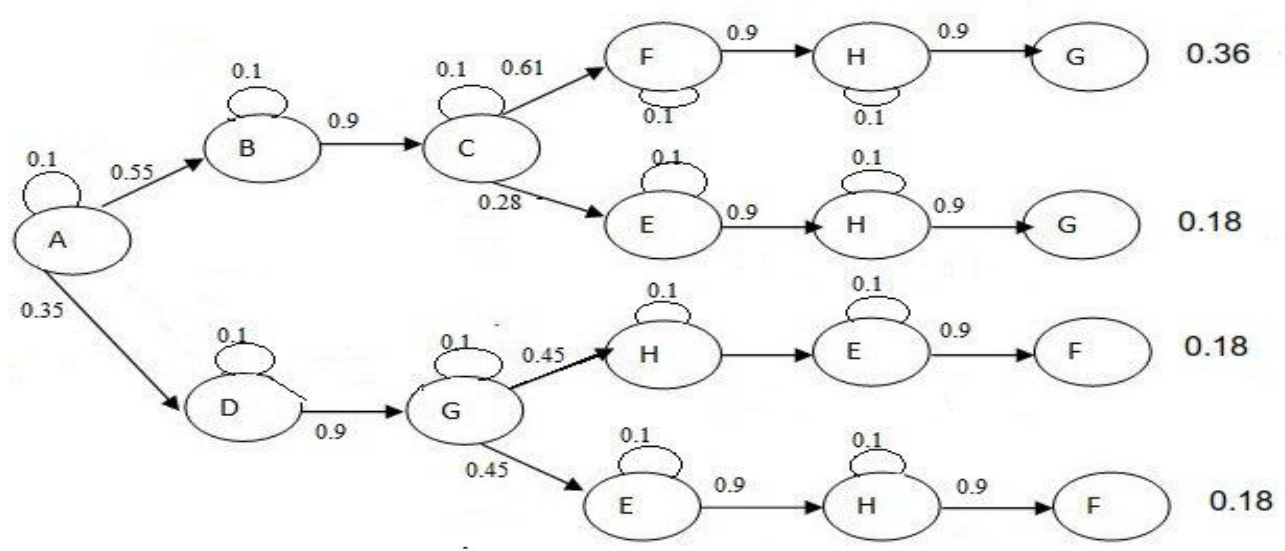

Figure 2. The Markov process scheme 


\subsection{Network selection}

In this step, the network selection part is presented; we'll discuss the parameters involved in the process and we'll explain the steps of the NS procedure. Subsequently, we propose an algorithm used to sort the available networks. The context of this work is to maintain the user best served with the best available RAT in case of high mobility. To do so, it is essential to minimize the number of vertical handover i.e., eliminating the unnecessary vertical handover to reduce the delay and PLR generated by this phenomenon. A vertical handover is the process where the mobile user changes the radio transmitter, or access media used to provide the requested services, while maintaining a predefined QoS. A mobile user in heterogeneous radio environments is under the coverage of several different networks with different characteristics: access medium, link layer...etc. (Benmammar and Krief, 2005). Therefore, the vertical handover decision coupled with the network selection process has become even more complex and crucial step towards achieving a seamless mobility in the fulfilment of the vision of being "Always Best Connected" (Keilson, 2012). Making the vertical handover only for the sake of maintaining connectivity during mobility is not the only objective; instead, the vertical handover process is coupled with the network selection process to get the maximum benefit from all available networks. Unnecessary vertical handover actually amplifies the signalling overhead and delay. It also forces the mobile user to consume more energy to achieve a successful VHO (Song and Jamalipour, 2005). To limit such negative effects on the QoS, we aim to minimize the number of vertical handover.

For starters, our proposed algorithm (Bendaoud et al., 2017) sorts the networks and provides the user with the best network. The algorithm is essentially based on the following equations:

$R_{i}=\sum_{j=1}^{m}$ income $_{i j}$

income $_{i j}=\left(\alpha-k_{i j}\right) * w[j]$.

$k_{i j}=\min \left(\operatorname{Vect}_{i j}\right)$.

Where:

$\alpha$ : is a fixed integer number equal to the number of alternatives.

$k_{i j}$ : it represents the rank order of the network $i$ for the criterion $j$.

Vect $_{i j}$ : is the column vector from the matrix mat where $j$ is fixed.

$w[j]$ : is the weight associated with the criterion " $j$ " for the alternative " $i$ ".

$i$ : is the alternative and $j$ is the criteria.

$\operatorname{mat}[n][m]$ : is the input matrix, it is represented by Table2. More details can be found in Algorithm 1, (Bendaoud et al., 2017).

We start by dividing the input matrix to a set of column vectors to get a group of vectors equal to the number of criteria.

The best case for a network ' $i$ ' is when it has the minimal value $R_{i}$ (we design the system to be a minimization problem) for the criterion ' $j$ ' this means the highest local ranking, thus $k_{i j}=0$ and the revenue is: $R_{i j}=\alpha * w[j]$. The worst case is when the network has the maximum value for the criterion ' $j$ ', i.e., $k_{i j}=\alpha-1$, i.e., the revenue is $R_{i j}=w[j]$.

The use of the weight concept is taken from the MADM approach. This concept allows us to give more sense to our objective function which ranks the networks based on their values for each criterion. The weight vector brings out the significant criteria and distinguishes them from the other less important criteria depending on the application requirements. Therefore, it assigns higher incomes to the corresponding networks. This strategy has the following advantages:

- Avoids the situation in which a network that has a good value for a non important criterion will have the same revenue as another network with a good value for an important criterion. This situation exists in the SAW method for example.

- The weight concept is the representation of application requirements in the system and that's how we distinguish between applications because each one has specific requirements. VoIP (Voice over IP) requires a minimum time delay and PLR for the video application. In addition, VoIP service requires also a good throughput. For the best-effort applications, the existing conditions are accepted, but the cost criterion is very important. This information is translated into digital values with the eigenvector method.

In this work, we used many parameters such as: cost, energy consumption, average throughput achieved, average delay, average PLR and network load. An example of the matrix is given in Table 3. These parameters displayed in the corresponding matrix are a margin values that the simulations produced. 


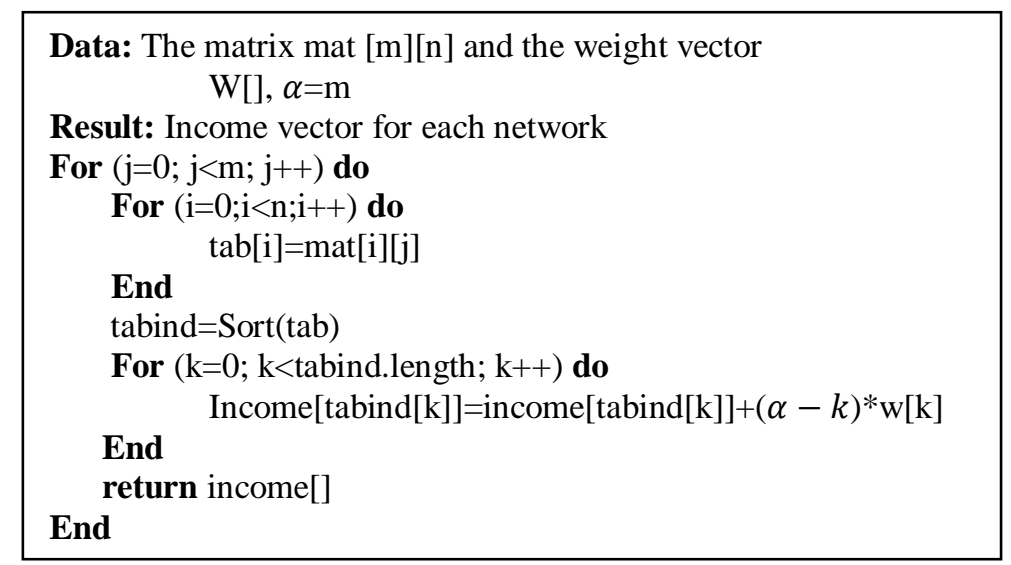

Algorithm 1: Modified-SAW function

The following steps (3 and 4) are vertical handover optimization oriented. A vertical handover VHO is a period of time in which the user is not connected to any RAT; in this tiny period of time there is no connection leading to some dropped packets which is known packet loss. Furthermore, these packet losses mean more delay to receive the data correctly. So, to eliminate these drawbacks, we added these two steps to try to get a configuration with an acceptable QoS without making many unnecessary vertical handover Figure3 ref $\{$ flowchart $\}$.

The objective of the steps three and four of the proposed architecture is to compare the performance of $C_{-}$init and $C_{-}$alt. The flowchart shows that in 2 from 3 cases, $C_{-}$init is selected as the best configuration for this path, and in the remaining case, the $C_{-}$alt is chosen.

\subsection{RATs configuration}

This section is the third step of the proposed framework in which we use the results of the network selection step to generate the configurations. A configuration is a list of successive RATs where each RAT is the best one on its zone. For example, we have three zones named A, B and C with three available RATs on each zone R1, R2 and R3. Ideally, the best configuration of RATs in these zones is the succession of the best RATs on each zone. One configuration amongst others for these zones could be selecting the same RAT on all the zones, which will erase the vertical handover caused by the user mobility. However, this choice (the same RAT on all the zones) is not necessarily the optimal configuration of RATs in term of the global QoS for the user. The objective of this part is to collect all the possible RATs configurations. In the upcoming section we will discuss the process of selection between the collected configurations.

For each path that the user is susceptible to take, we make an off-line network selection to get the RATs configurations. The obvious one is the combination of the best RAT for each zone which constitutes a given path which is named the initial configuration. Moreover, we generate also the alternative configurations. Conversely, selecting the initial configuration might introduce multiple vertical handovers. Essentially, our idea aims to generate the alternative configurations that minimize or eliminate the vertical handover occurrences. Generally, there are two alternative configurations as we are able to change a RAT with another RAT which has the next highest score in RAT ranking of each zone.

When having the initial and alternative configurations, it is possible to compare the alternative configurations with the initial one based on the delay, Packet Loss Rate (PLR) and especially adding the jitter caused by the VHOs. We choose these parameters as they are the most essential ones for a best performance during the service session. The evaluation function depends also on the service application. Indeed, for the VoIP service, the delay is more important than packet loss. Same for the video service, these parameters have a similar importance. In the following, a configuration is represented by $C$, so the initial configuration is named $C_{\text {_ }}$ init and $C_{-}$alt for the alternative configurations.

We remember that the vertical handover latency is the duration from the handover initiation to the handover completion which is equal to the amount of time from when the mobile is disconnected from the older RAT to the time when it receives the first packet from the new RAT. The transmission delay and the handover delay are perceived by the mobile user, it is well known that the transmission delay is the amount of time taken by the packet to reach from source to destination; it is the sum of transmission delay, propagation delay and processing delay (Payaswini and Manjaiah, 2014). These delays are summed to give us the end to end delay (e2e). The VHO latency is modelled in our situation as jitter, indeed, when a VHO occurred, the user perceives (a variation) surely an augmentation of the transmission 
delay which represents the jitter. The challenge then is whether to select the initial configuration which has the suited values of delay and PLR while it is mostly susceptible to generate jitter due to the possible VHO or, to select an alternative configuration with higher delay and PLR but without any jitter because of the absence of any VHO. These different metrics have a common influence on the mobile user which is the deterioration of the QoS for the user so; we will try to connect the user with the configuration which generate the lesser degradation of QoS.

An alternative configuration must minimize the vertical handover occurrences, i,e., the number of vertical handovers in the alternative configuration must be lesser than the number of vertical handover in the initial one. To select one configuration among others to be used for one path, we use the formulas 7,8 and 9 . Whereas, if the formulas 7 and 8 are satisfied, we select the $C_{X}$ otherwise, we select the $C_{Y}$, where:

C_X[i].delay and C_X[i].plr: They represent the value of delay and PLR for the zone ' $\mathrm{i}$ ' of the alternative $\mathrm{X}$.

nb_VHO_(C_X): This parameter means the number of vertical handover in the configuration C_X. In other words, it is simply the number of RATs in which, the present and the previous RAT are different. VHO_delay and VHO_plr: Are the latency and the PLR introduced when the vertical handover occurs. Both of these values are extracted from previous related work (Fettouh et al., 2013; Payaswini and Manjaiah, 2014; Nithyanandan and Parthiban, 2012).

$\mathrm{C}_{\text {alt }[\mathrm{i}]}$. delay $\leq 150 \mathrm{~ms}$.

$\mathrm{C}_{\text {init }[\mathrm{i}]}$. delay $+\mathrm{VHO}_{\text {delay }}>150 \mathrm{~ms}$.

$\mathrm{C}_{\mathrm{alt}[\mathrm{i}]}$. delay $-\mathrm{C}_{\mathrm{alt}[\mathrm{i}-1]}$. delay $<|30| \mathrm{ms}$.

These equations are applied for each zone. Where ' $i$ ' represents one zone, $150 \mathrm{~ms}$ represents the maximum value of delay tolerated for the real time applications. $30 \mathrm{~ms}$ is the maximum jitter tolerated and beyond it the QoS will be affected. The previous equations focuses only on the delay, because based on (Fettouh et al., 2013; Payaswini and Manjaiah, 2014; Nithyanandan and Parthiban, 2012) the PLR resulted from a VHO is in the range of $2 \%$ which is insignificant, so we have ignored the PLR in our process. For each path among the predicted ones, these equations 7, 8 and 9 are used to select only one configuration and to eliminate the other configurations. At the end of this step, we get one configuration of RATs and the best configuration is considered. In the next section, we are going to propose a process that will take only one configuration to be used for the entire predicted paths.

\subsection{Selection of the adequate configuration}

The final step of the process is to choose one configuration to be used in the entire predicted paths. This configuration is probably the best suited for the whole session duration. To do that, the idea is to take all the zones from $Z$ and for each one of them; we select the appropriate RAT using the algorithm 2. $Z$ is the list which contains the zones susceptible to be visited by the mobile user. For each path $\boldsymbol{p}_{\boldsymbol{i}}$ we have a configuration $C_{-} p i$ (see step three of the process).

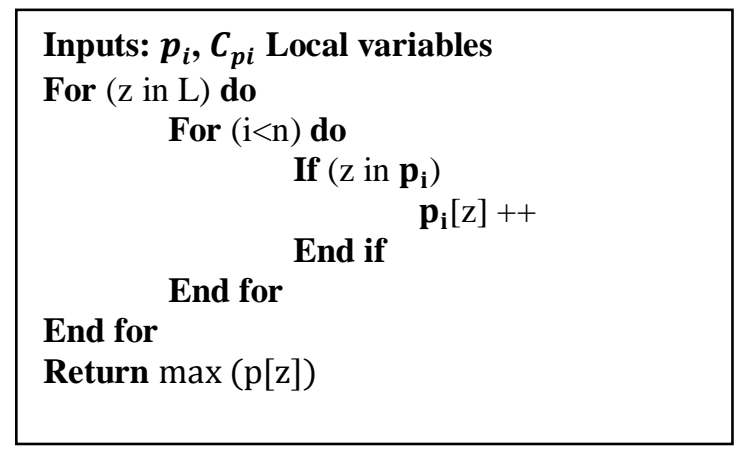

Algorithm 2: performance function

The algorithm 2 takes as inputs the predicted paths and the selected configuration of RAT in each path, the output is a zone with a RAT. The process consists of selecting all zones which are susceptible to be visited by the mobile user and associate to those zones the RAT which have been selected by the maximum number of paths. In the following we make a recap of the proposed methodology and, give the general algorithm that summarizes the described process. 
The internal process of Algorithm 3 is composed of four steps:

- The first step is the path prediction. We used in this step the order-2 Markov process. The algorithm takes as inputs two parameters: Mob-position and Loc-history which are the mobile position and the location history of this mobile user. The result of this step is the combination of paths $c h_{i}$ and their probabilities $p r b_{i}$.

- The second step consists in the making of the network selection on the predicted paths. In this step we make a network selection on each zone of each predicted path. We start by creating the matrix system for each zone in which, the rows are the RATs and the columns are the criteria like bandwidth, delay, PLR, energy consumption and cost. The result of this step is a matrix $R k_{i}[][]$ that contains the ranking order of RATs for each zone on the path $c h_{i}$.

- The third step, we collect the configurations and we select only one configuration for each path. First, the initial configuration that we generate is a trivial one because we just take the RATs with the first ranking order. The alternative configurations are a mix between the RATs with the first ranking order and those with the second ranking order in which, the number of vertical handover is minimized. After collecting these configurations, the objective of the next step is to select only one configuration for each path and to eliminate the others using the equations 7,8 and 9.

- In the final step, we create a new configuration called the adequate configuration which contains on each zone the RAT which has been selected by the maximum number of paths.

\section{Evaluation performance}

We consider the same example presented earlier in Figure 1. Supposing that the mobile user is in the cell $a$, the first step is to use the $\mathrm{O}(2)$ Markov model function to get the possible paths that the user can take with their probabilities. Hypothetically, one of the paths predicted is $\boldsymbol{c h}_{\boldsymbol{i}}={ }^{\prime \prime} a->b->c->e->h^{\prime \prime}$ with a given probability. The next step is to make the off-line network selection on all the zones constituting the path $\boldsymbol{c h} \boldsymbol{h}_{\boldsymbol{i}}$. The first task of the step 2 in Algorithm 3 is to generate the input matrix Table 3 based on the Table 2 .

The values presented in Table 3 are generated randomly based on Table 2 which contains range values for each criterion generated using NS3 simulator (Network simultor 3, 2011). Other works have provided the same QoS values for these networks (Grigorik, 2013; Hasib, 2006).

Furthermore, when we execute the step 2 of Algorithm 3, we make the network selection. Algorithm 1 takes as an input the data matrix and a weight vector that represents the service application (voice, video or web navigation). The network selection results are presented in the Table 3.

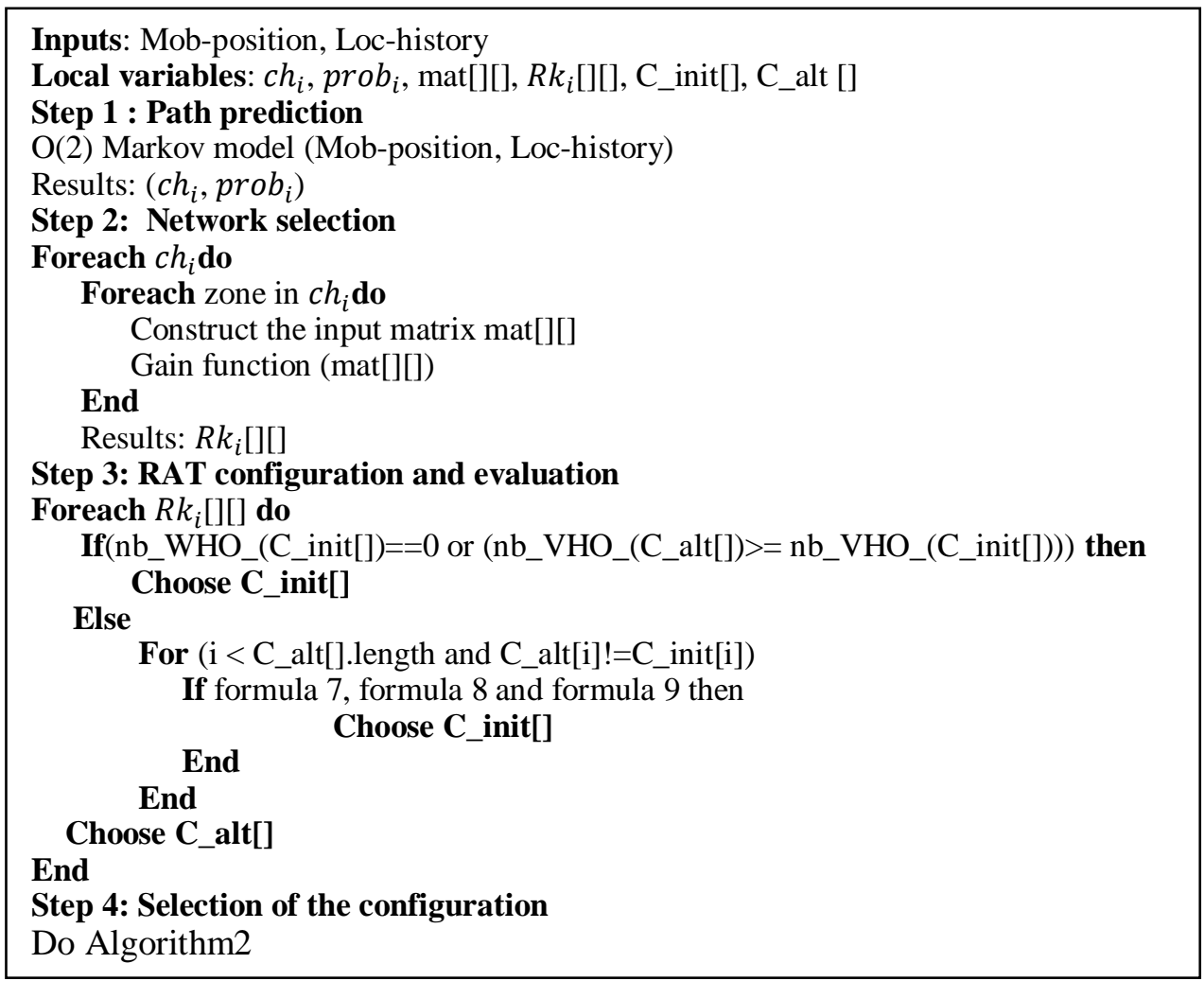

Algorithm 3: Mobility-aware network selection proposal 
The following flowchart brings clarifies more the proposed network selection process. It helps us to understand the whole process.

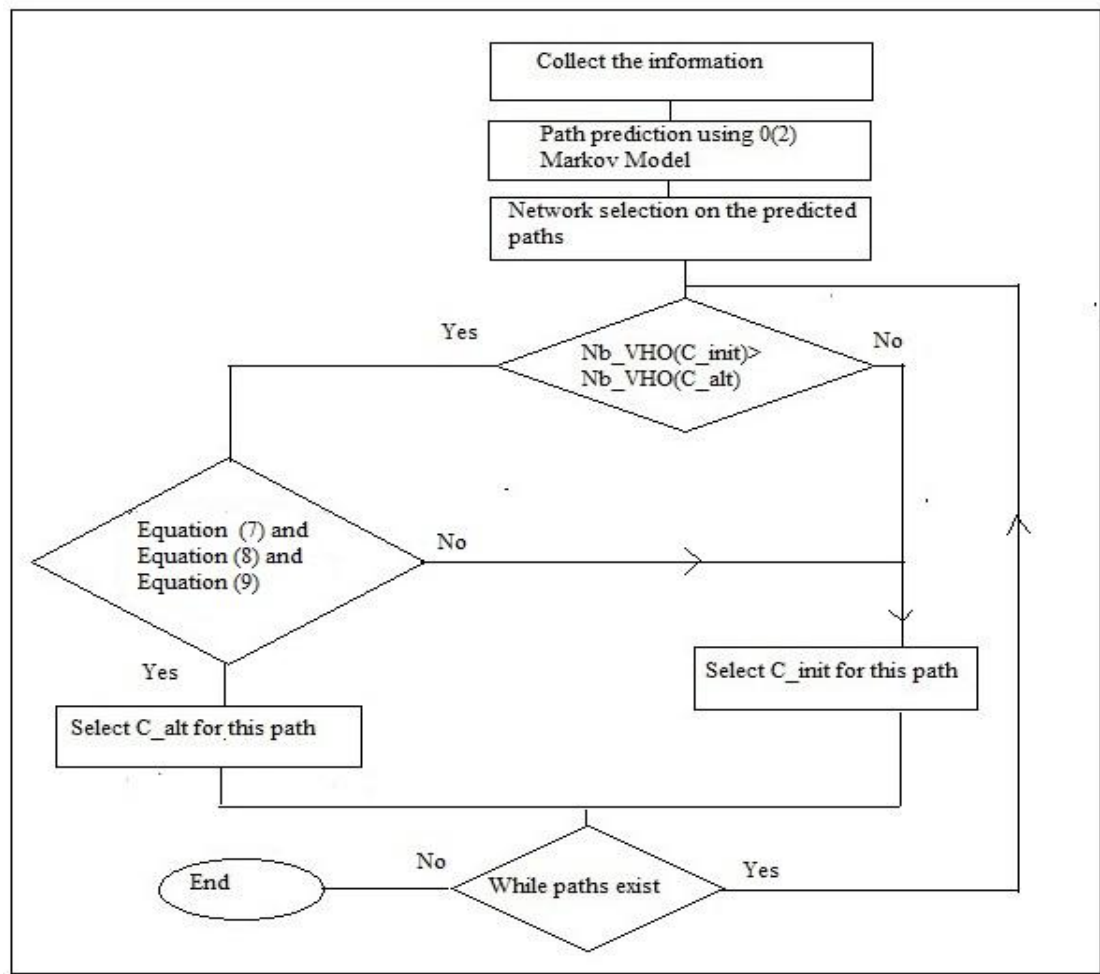

Figure 3. The proposed flowchart

Table 2. The matrix model

\begin{tabular}{|c|c|c|c|c|c|}
\hline & Bandwidth & Delay (ms) & PLR (\%) & Energy & Cost \\
\hline Wi-Fi & $1-11$ & $100-150$ & $0.2-3$ & - & 1 \\
\hline 3G & $1-14$ & $25-50$ & $0.2-3$ & - & 5 \\
\hline LTE & $1-100$ & $60-100$ & $0.2-3$ & - & 2 \\
\hline
\end{tabular}

Table 3. The input matrix of the zones A, B, C, E, H

\begin{tabular}{|c|c|c|c|c|c|c|}
\hline Zones & RATs & Thr (Mb/s) & Delay $(\mathrm{ms})$ & PLR (\%) & Energy (J) & Cost \\
\hline Zone 'A' & $\mathrm{R} 0$ & 10.86 & 115.54 & 1.59 & 5.33 & 0.2 \\
& $\mathrm{R} 1$ & 4.42 & 31.23 & 1.23 & 4.44 & 1.0 \\
& $\mathrm{R} 2$ & 35.46 & 80.26 & 2.55 & 51.94 & 0.4 \\
\hline Zone 'B' & $\mathrm{R} 0$ & 9.61 & 103.87 & 1.71 & 4.76 & 0.2 \\
& $\mathrm{R} 1$ & 6.09 & 30.63 & 2.62 & 5.69 & 1.0 \\
& $\mathrm{R} 2$ & 32.46 & 82.80 & 1.44 & 51.09 & 0.4 \\
\hline Zone 'C' & $\mathrm{R} 0$ & 8.47 & 120.51 & 2.13 & 4.19 & 0.2 \\
& $\mathrm{R} 1$ & 1.82 & 40.81 & 2.93 & 2.48 & 1.0 \\
& $\mathrm{R} 2$ & 50 & 79.95 & 1.63 & 61.83 & 0.4 \\
\hline Zone 'D' & $\mathrm{R} 0$ & 4.52 & 120.20 & 2.72 & 2.33 & 0.2 \\
& $\mathrm{R} 1$ & 4.78 & 40.19 & 1.12 & 4.71 & 1.0 \\
& $\mathrm{R} 2$ & 41 & 68.11 & 2.84 & 53.74 & 0.4 \\
\hline Zone 'E' & $\mathrm{R} 0$ & 10.52 & 102.83 & 2.89 & 5.16 & 0.2 \\
& $\mathrm{R} 1$ & 8.13 & 41.54 & 2.13 & 7.22 & 1.0 \\
& $\mathrm{R} 2$ & 30.5 & 69.10 & 2.08 & 39.84 & 0.4 \\
\hline
\end{tabular}

\subsection{Validation of the proposed scheme}

So, before we verify if our selection process is more efficient than the other existed ones, we must present an example to explain clearly the process and how it works. The $C_{-}$init is the configuration containing the best RAT for each zone, in our example, $C_{-}$init $=[R 1, R 2, R 2, R \bar{l}, R 1]$. This configuration 
offers the best QoS for the user at each zone of the path $\boldsymbol{c} \boldsymbol{h}_{\boldsymbol{i}}$. Nonetheless, this implies that the user has to make 2 vertical handover which could cause the decline of the QoS of the entire path i.e., the entire session. The alternative configurations are selected from the second row of the results Table 4. We have chosen the second row because generally the networks of this row have acceptable QoS performances in comparison with the ones in the third row. So, based on $C_{-}$init and the second row of the Table 4, we get two alternative configurations. The first one is $C 1 \_$alt $=[R 1, R 1, R 1, R 1, R 1]$ and the second one is $C 2 \_$alt $=[R 2, R 2, R 2, R 2, R 2]$. Remember that each $C \_$alt must minimize the number of vertical handover; Hence, we cannot accept any alternative configuration which has a number of vertical handover greater than or equal to the number of vertical handovers in $C_{-}$init. Previous works revealed that making a vertical handover introduces additional delay of $86 \mathrm{~ms}$ and PLR of $3 \%$ of packets sent in the period of vertical handover (Magagula et al., 2010). The authors in (Shen et al., 2010) have provided a vertical handover latency in the range of 50 to $100 \mathrm{~ms}$ and a packet loss rate range from $1 \%$ to $3 \%$. So, we have decided to take an average value based on those references which means, a vertical handover latency of $50 \mathrm{~ms}$ and a packet loss rate equal to $2 \%$. These values are used later in our process.

From Table 3 we can see and affirm that for zone A, R1 is more suited than R2 (lesser delay and PLR), so C1_alt is the alternative configuration which will be compared to the $C$ init configuration. Thus, the selection of the best configuration in the third step (Algorithm 3) is based on reducing the number of vertical handovers while ensuring a good QoS for the user. Otherwise, the initial configuration is kept. The comparison between the configurations is performed in term of the number of vertical handovers and the QoS offered to the users. When comparing the $C$ _init and $C 1$ alt, we confirm that C1_alt has no vertical handover. Thus, it satisfies the first condition. Secondly, we see if the changes of RATs in the second and third positions of the $C_{-}$init don't degrade the QoS or at least give a similar performance values.

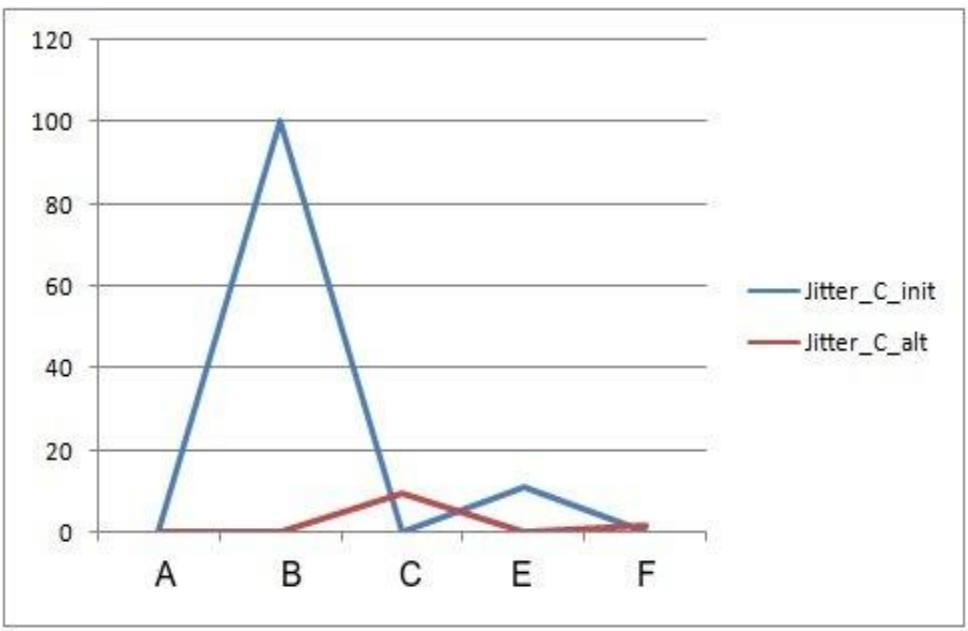

Figure 4. Jitter comparison C_init vs C_alt (ms)

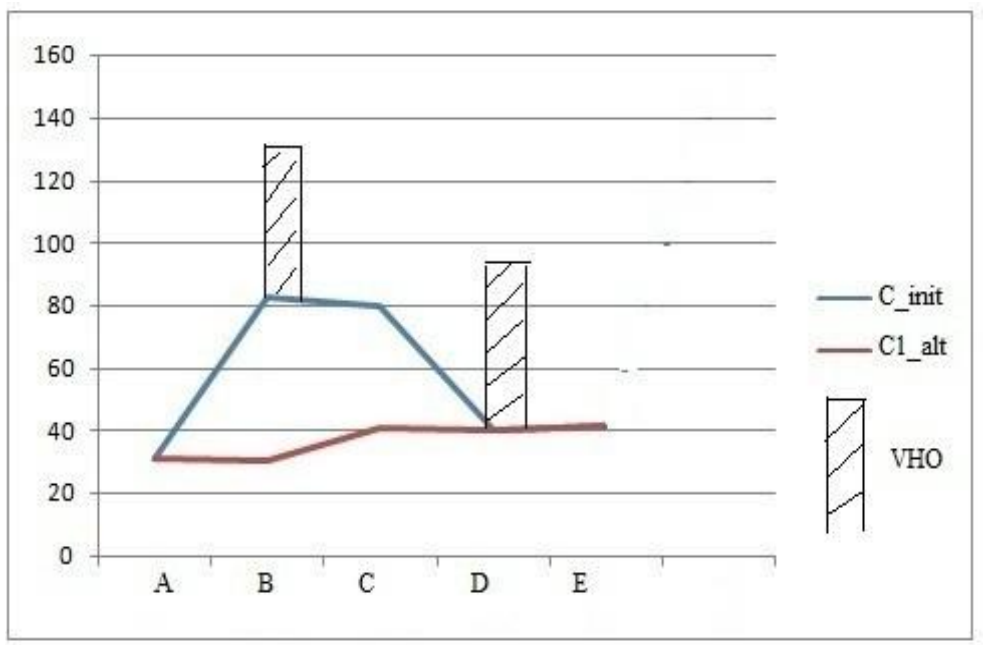

Figure 5. Instant delay comparison C_init vs C_alt (ms) 
For our example, we have to compare C_init with C_alt. The network selection step has selected the $\mathrm{C}_{-}$init as the suited configuration for the user. The steps 3 and 4 consist on making some deep verification. In C_init we have two VHO, these 2 VHO affect the QoS of the user, because in this lapse of time (the VHO time) there is no connection, which means some packets will be lost and others will have more delays. Thus, the QoS in this lapse of time will be affected. So, the goal of this study is to minimize the VHO occurrence especially when it is unnecessary. So, we have added the delays and PLR caused by the VHO to the transmission delay and PLR. In this example, we have concluded that the C_alt is more suited but in other cases it might be not.

Figure 5 shows that $\mathrm{C} 1$ _alt produces a lower delay by comparison with $\mathrm{C} \_$init or at least the same delay. In addition, we have two VHO in C_init which generates more latency. We remark that $\mathrm{C}_{-}$init provides higher jitter more than the tolerated values, Figure 4.

If we select the network R2 in zone B, the user will have more variations in delay and packet loss which is known as jitter. Thus, with this information we can say that choosing R1 instead of R2 is the right choice for the user. From this, we can say that $C_{-}$alt is better than $C_{-}$init. As a result, it will be wiser for the user to not choose the initial configuration. Now, we must compare C_alt and C1_alt.

The Figure 4 shows that R1 is better than R2 in terms of QoS in the zone 'B' and ' $\mathrm{C}$ ' and it has the same performance on the other zones 'A', 'E' and 'H'. From this we conclude that the best configuration for the user is definitely C_alt because it eliminates the vertical handover and do not degrade the QoS for the user in all the zones of the path because it presents a low jitter.

The Figure 4 represents the instant delay for both configurations C_init and C_alt, the figure shows clearly that choosing the alternative configuration provides a stable and lesser delay without big variation in comparison with the initial configuration which provides a larger delays at each zone with higher jitter.

The same process must be performed for the other possible paths. Indeed, for each selected configuration, we apply the proposed process to choose the best configurations and using it for any chosen path.

The results of this example show clearly that our selection mechanism improves the performance. Indeed, for each path, we get the best configuration that minimizes the unnecessary vertical handover while we maintain a good QoS.

\subsection{Comparison with other works}

After we have presented an example of the proposed framework, it is time to compare our proposal with the existed works.

Table 4. The network selection results for the path $\boldsymbol{c h}$

\begin{tabular}{|c|c|c|c|c|c|}
\hline & A & B & C & E & R1 \\
\hline Proposal & R1 & R2 & R1 \\
& R2 & R2 & R1 & R0 \\
& R0 & R1 & R0 & R0 & R0 \\
& R0 & R0 & R0 & R1 \\
\hline TOPSIS [10] & R2 & R2 & R2 & R0 & R2 \\
& R1 & R1 & R0 & R2 \\
& R2 & R2 & R1 & R0 \\
\hline WPM [14] & R0 & R2 & R1 & R2 & R1 \\
\hline
\end{tabular}

The results in Table 4 provide the ranking order of RATs from zone ' $A$ ' to zone ' $E$ ' using different methods, in the following, we will compare these results.

For zone ' $A$ ', our proposal ranks the RATs like R1, R2, and R0. The other methods give other ranking orders such as TOPSIS that ranks the RATs as R0, R2, R1; WPM and AHP give a ranking list equal to R2, R0, R1 for the same zone $A$.

We will compare the QoS offered by R0, R1 and R2 for all zones to know which method allows the suitable selection of RATs. 


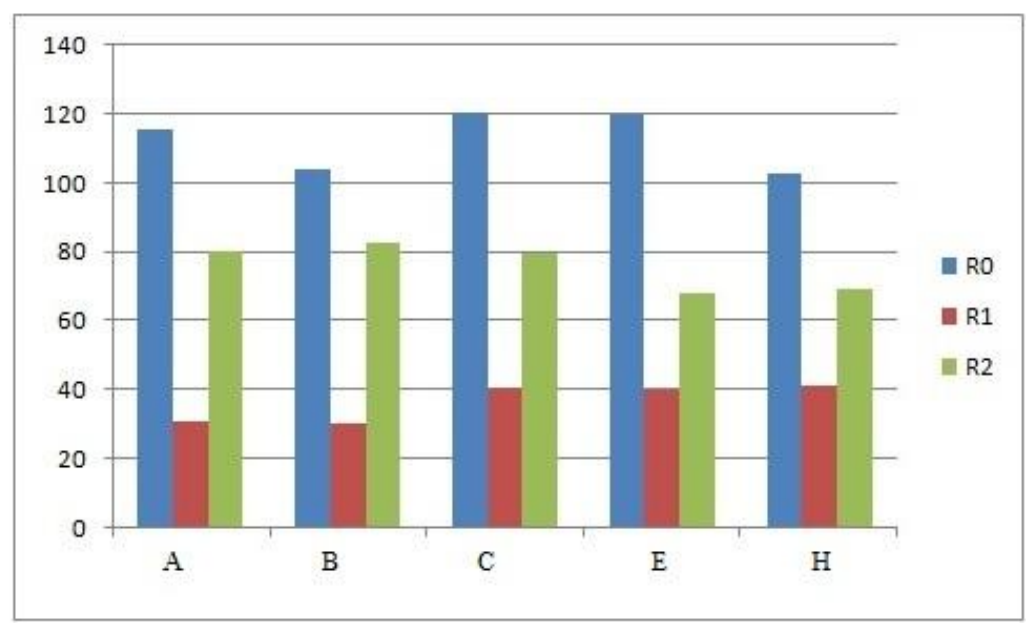

Figure 6. Delay of R0, R1 and R2 for each zone (ms)

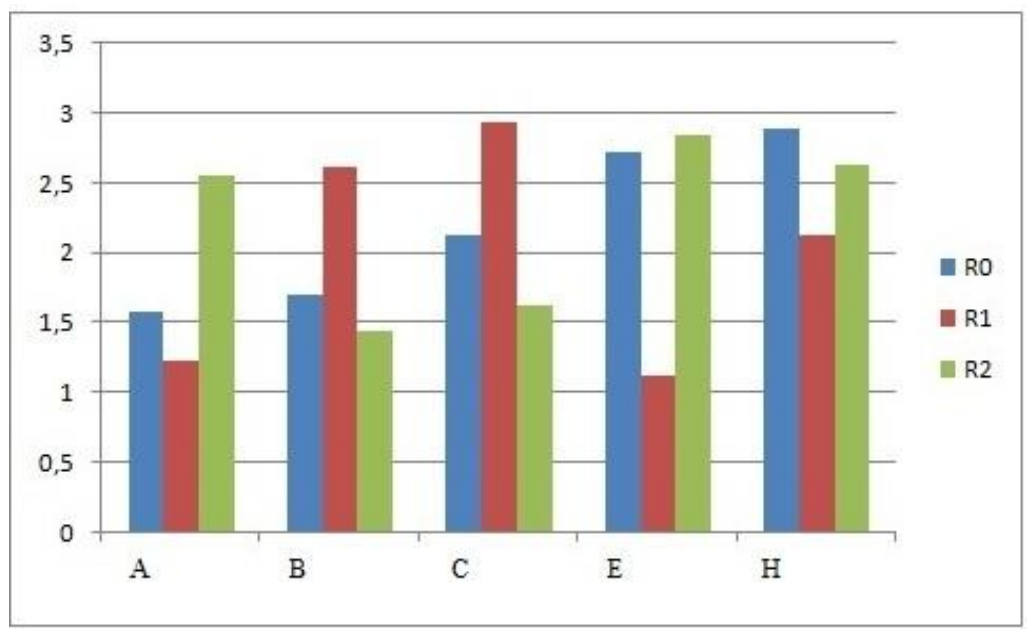

Figure 7. PLR of R0, R1 and R2 for each zone (\%)

From Figure 6, Figure 7 and Table 4, we deduce that our proposal is more suited for the users and gives more accurate ranking of RATs which provides a better QoS. In zone ' $A$ ' for example, our proposal selects R1 as the appropriate RAT. Whilst, TOPSIS chooses R0, WPM and AHP select R2. Figures (6 and 7) show that R1 is more suited in zone ' $A$ ' and so on for the other zones.

From these figures, we conclude that our selection mechanism offers the accurate ranking order for the RATs. These methods such as TOPSIS, WMP and AHP have been used by several works like (Savitha and Chandrasekar, 2011a; TalebiFard and Leung, 2011; Shen et al., 2010). In the Table 5, we present the advantages and inconvenient of some important works in this field.

Table 5. Theoretical comparison

\begin{tabular}{|l|l|l|}
\hline \multicolumn{1}{|c|}{ Work } & \multicolumn{1}{|c|}{ Advantages } & \multicolumn{1}{c|}{ Inconvenient } \\
\hline $\begin{array}{l}\text { Paper } \\
\text { (Hasib, 2006) }\end{array}$ & Reducing the end delay & $\begin{array}{l}\text { Only 802.11n networks } \\
\text { A queue with packet prioritization } \\
\text { No consideration of handover }\end{array}$ \\
\hline $\begin{array}{l}\text { Paper } \\
\text { Nguyen-Duc and } \\
\text { Kamioka, 2016) }\end{array}$ & Offering the required QoS & $\begin{array}{l}\text { Many correlated parameters } \\
\text { Only RSS to model the mobility } \\
\text { No consideration of handover }\end{array}$ \\
\hline $\begin{array}{l}\text { Paper } \\
\text { Wang and Binet, 2009) }\end{array}$ & $\begin{array}{l}\text { Prediction of mobility using Markov model } \\
\text { Consideration of the handover }\end{array}$ & $\begin{array}{l}\text { No network selection in the process } \\
\text { Only WLAN and UMTS }\end{array}$ \\
\hline Our approach & $\begin{array}{l}\text { Consideration of the handover } \\
\text { Reducing the end delay }\end{array}$ & $\begin{array}{l}\text { Many steps mean more time to get the optimal } \\
\text { configuration of RATs } \\
\text { Probabilistic model }\end{array}$ \\
\hline
\end{tabular}


In this section we have displayed the originality of our proposal with an example. In this example, any other QoS improvement oriented method chooses the initial configuration which invoke more unnecessary vertical handovers. However, our intelligent approach allows the user to have a high QoS while minimizing the vertical handover occurrences in the system. As a comparison with other works, we haven't find works using the same approach. In the Table 5, we give a comparison between our proposal and other works.

\section{Conclusion}

The objective of this study was to propose a network selection in the case of high mobility. The proposed approach begins with the path prediction using Markov model order 2. We used the latter because it was proven that this predictor provides the best performance for our case study. Subsequently, we apply a network selection across the zones of the paths predicted, we have proved that our selection mechanism performs very well and outperforms other previous proposals. The third step is the evaluation of the RATs configuration and finally, we use the mean value to get the configuration most adapted with all the predicted paths. This approach gives the user a lesser number of vertical handover while it maintains a good or at least an acceptable QoS. The proposed scheme allows the user to make a more realistic scenario for network selection in a heterogeneous environment; the strength of this study resides in the consideration of the mobility as an environment for the users which is the right way to treat this problem. In the contrary, other studies consider the mobility as a simple input in the matrix process to do the selection which is incorrect in reality. We believe that this study is more complete and it is the only way to make a correct network selection in a heterogeneous environment. In the same time, the results depend on the prediction accuracy of the paths which is a drawback for this proposal.

As a perspective, it will be interesting study the computation time of the whole process and try to enhance the prediction model to swap from a probabilistic model to a certitude model; also, we hope been able to realize a real implementation of the proposal.

\section{References}

1. Bendaoud, F., Abdennebi, M. and Didi, F. (2018) Network Selection in Wireless Heterogeneous Networks: a Survey. In: Journal of Telecommunications and Information Technology, 4, p. 64.

2. Yafang, W., Huimin, C. and Jinyan, Z. (2010) Notice of retraction network access selection algorithm based on the analytic hierarehy process and gray relation analysis. In: New Trends in Information Science and Service Science (NISS), 4th International Conference, 2010, IEEE, pp. 503-506.

3. Bari, F. and Leung, V. (2007) Multi-attribute network selection by iterative topsis for heterogeneous wireless access. In: 4th IEEE Consumer Communications and Networking Conference, pp. 808-812.

4. Bendaoud, F., Abdennebi, M., and Didi, F. (2015) Network selection using game theory. In: Control, Engineering \& Information Technology (CEIT), 3rd International Conference, IEEE, pp. 1-6.

5. Lahby, M., Baghla, S. and Sekkaki, A. (2015) Survey and comparison of MADM methods for network selection access in heterogeneous networks. In: New Technologies, Mobility and Security (NTMS), 7th International Conference, IEEE, pp. 1-6.

6. Fu, J., Wu, J., Zhang, J., Ping, L. and Li, Z. (2010) A novel AHP and GRA based handover decision mechanism in heterogeneous wireless networks. In: Information computing and applications, Springer, pp. 213-220.

7. Shaikh, F.S. (2010) Intelligent proactive handover and QoS management using TBVH in heterogeneous networks. $\mathrm{PhD}$ thesis, Citeseer.

8. François, J.-M. and Leduc, G. (2006) Prédiction de mobilité par le mobile ou par le point d'accès: comparaison sur base de traces réelles. In: Colloque Francophone sur l'Ingénierie des ProtocolesCFIP 2006, Hermès, pp. 12-p.

9. Song, L., Kotz, D., Jain, R. and He, X. (2004) Evaluating location predictors with extensive Wi-Fi mobility data. In: INFOCOM 2004. Twenty-third AnnualJoint Conference of the IEEE Computer and Communications Societies, 2, pp. 1414-1424.

10. Savitha, K. and Chandrasekar, C. (2011a) Trusted network selection using saw and topsis algorithms for heterogeneous wireless networks, arXiv preprint arXiv:1108.0141.

11. Nguyen-Vuong, Q.-T., Ghamri-Doudane, Y. and Agoulmine, N. (2008) On utility models for access network selection in wireless heterogeneous networks. In: Network Operations and Management Symposium, 2008. NOMS 2008. IEEE, pp. 144-151. 
12. Salih, Y. K., See, O. H., Ibrahim, R. W., Yussof, S. and Iqbal, A. (2015) A user-centric game selection model based on user preferences for the selection of the best heterogeneous wireless network. Annals of telecommunications-annales des télécommunications, 70(5-6), pp. 239-248.

13. Savitha, K. and Chandrasekar, C. (2011b) Vertical handover decision schemes using sawand WPM for network selection in heterogeneous wireless networks, arXiv preprint arXiv:1109.4490.

14. TalebiFard, P. and Leung, V. C. (2011) Context-aware mobility management in heterogeneous network environments, JoWUA, 2(2), pp. 19-32.

15. Gambs, S., Killijian, M.-O. and del Prado Cortez, M. N. (2012) Next place prediction using mobility markov chains. In: Proceedings of the First Workshop on Measurement, Privacy, and Mobility, ACM, p. 3.

16. Fettouh, A., El Kamoun, N. and El Fazziki, A. (2013) Improving vertical handover performance of real time applications over heterogeneous wireless networks, International Journal of Computer Applications, 62(21).

17. Magagula, L.A., Chan, H.A. and Falowo, O.E. (2010) PMIPv6-HC: Handover mechanism for reducing handover delay and packet loss in NGWN. In: Global Telecommunications Conference (GLOBECOM 2010), 2010 IEEE, pp. 1-5.

18. Roman, C., Liao, R., Ball, P. and Ou, S. (2016) Mobility and network selection in heterogeneous wireless networks: User approach and implementation, Network Protocols and Algorithms, 8(2), pp. $107-122$.

19. Wang, L. and Binet, D. (2009) Madm-based network selection in heterogeneous wireless networks: A simulation study. In: Wireless Communication, Vehicular Technology, Information Theory and Aerospace \& Electronic Systems Technology, 2009. Wireless VITAE 2009. 1st International Conference, IEEE, pp. 559-564.

20. Benmammar, B. and Krief, F. (2005) Gestion dynamique du handover horizontal et vertical basée sur le profil de mobilité de l'utilisateur. In: Colloque GRES 2005: Gestion de REseaux et de Services.

21. Keilson, J. (2012) Markov Chain Models - Rarity and Exponentiality, Springer Science \& Business Media, 28.

22. Song, Q. and Jamalipour, A. (2005) An adaptive quality-of-service network selection mechanism for heterogeneous mobile networks, Wireless Communications and Mobile Computing, 5(6), pp. 697-708.

23. Gustafsson, E. and Jonsson, A. (2003) Always best connected, IEEE Wireless Communications, 10(1), pp. 49-55.

24. Choi, H.-H. and Cho, D.-H. (2005) Takeover: a new vertical handover concept for nextgeneration heterogeneous networks. In: Vehicular Technology Conference, 2005. VTC 2005-Spring. 2005 IEEE 61 st, 4, pp. 2225-2229.

25. Bendaoud, F., Didi, F. and Abdennebi, M. (2017) A modified-saw for network selection in heterogeneous wireless networks, ECTI Transactions on Electrical Engineering, Electronics, and Communications, 15(2), pp. 8-17.

26. Payaswini, P. and Manjaiah, D. (2014) Simulation and performance analysis of vertical handoff between WiFi and WIMAX using media independent handover services, International Journal of Computer Applications, 87(4).

27. Nithyanandan, L. and Parthiban, I. (2012) Vertical handoff in WLAN-WIMAX-LTE heterogeneous networks through gateway relocation, International Journal of Wireless \& Mobile Networks, 4(4), p. 203.

28. Network simultor 3, https://www.nsnam.org/, 2011.

29. Grigorik, I. (2013) High Performance Browser Networking: What every web developer should know about networking and web performance. O'Reilly Media, Inc.

30. Hasib, M. (2006) Analysis of packet loss probing in packet networks. PhD thesis, Queen Mary, University of London.

31. Nguyen-Duc, T. and Kamioka, E. (2016) An energy-efficient mobile-controlled vertical handover management for real time services, Journal of Computer and Communications, 4(17), p. 59.

32. Shen, D.-M., Tian, H. and Sun, L. (2010) The QoE-oriented heterogeneous network selection based on fuzzy AHP methodology. In: Proc. of UBICOMM2010 (The Forth International Conference on Mobile Ubiquitous Computing, Systems, Services and Technologies), pp. 275-280. 